\title{
Experimental Investigation of Corner Flows in Rectangular Supersonic Inlets with 3D Shock-Boundary Layer Effects
}

\author{
W. Ethan Eagle ${ }^{1}$ and James F. Driscoll ${ }^{2}$ \\ University of Michigan, Ann Arbor, MI, 48109 \\ John A. Benek ${ }^{3}$ \\ U.S. Air Force Research Laboratory, Wright-Patterson Air Force Base, OH, 45433
}

\begin{abstract}
In a rectangular supersonic inlet the first oblique shock wave will interact with both the sidewall boundary layers and with the corner flows. This can create large, complex 3-D separation zones that reduce the effective flow area and can lead to the unstart of the inlet. Experiments were conducted in the Michigan Glass Wind Tunnel at Mach 2.75 and at Mach 2.0 to quantify these flow separation patterns. Video was recorded of the unsteady formation of separation zones as the inlet starts. Oil streak patterns and Schlieren images indicate that the downward flow caused by the oblique shock forces some of the flow near the corner to move upstream. This flow moving upstream in the corner creates a sidewall separation bubble, which significantly deflects the free stream upstream of the shock wave. Using a six-degree wedge, a "small" corner flow-shock boundary layer interaction (CF-SBLI) separated region was created which was found to be optimum for CFD simulation. Using a ten-degree wedge generated a stronger shock and a "large" CF-SBLI separated region, but the flow area was reduced so much that the inlet was too close to unstart to allow for stable experimental measurements or CFD simulation.
\end{abstract}

\section{Introduction and Background}

$1_{\text {NCIDENT oblique shocks }}^{[1,2]}$, compression corners ${ }^{[3,4]}$, swept fins ${ }^{[4-7]}$, and normal shocks ${ }^{[8-10]}$ generate pressure rises which result in thickening or separation of the boundary layer. Unfortunately, because each flow has different characteristic scales, these flows defy a simple two-dimensional analytic parameterization; further difficulty regarding understanding of shock-foot unsteadiness is still a matter of concern ${ }^{[4,11-13]}$.

An effort sponsored by the Air Force Collaborative Center for Aeronautical Sciences (AF-CCAS) is underway investigating shock wave boundary layer interaction (SBLI) for applications in the design of next-generation high performance (possibly bleed-less) engine inlets. The effort thus far includes benchmark quality stereo particle image velocimetry (SPIV) data of a single oblique shock interaction. CCAS has developed an experimental database for CFD assessment. Thus measurements from this experiment not only offer a chance for better physical understanding of SBLI, but thanks to careful experimental design and documentation, also enable validation of advanced CFD codes. A CCAS-sponsored workshop on SBLI was held in January 2010 at the Orlando AIAA Aerospace Sciences Meeting, where the first round of CFD validation and comparison to data was completed ${ }^{[14]}$. The meeting concluded with a discussion of the future of this effort and focused on what work is still needed in the area.

A new set of criteria were developed to enhance the effort that would move towards an ' $3-\mathrm{D}$ inlet design' investigation rather than a single 2-D shock-boundary layer 'unit physics' problem ${ }^{[15]}$. One problem arising in realistic inlet geometries, but commonly ignored by the 'unit physics' approach, is the effect of flow field threedimensionality. Previous results from our experiment have called into question our assumptions of boundary layer behavior in the corner regions. The current experiment adds to our present understanding by visualizing the cause and effect of corner induced three-dimensionality in an oblique shock boundary layer interaction at different shock strengths.

${ }^{1}$ Graduate Research Assistant, Aerospace Engineering Department, 1320 Beal Avenue \#2004 FXB Ann Arbor, Michigan 48105, AIAA Student Member.

${ }^{2}$ Professor, Aerospace Engineering Department, AIAA Fellow.

${ }^{3}$ Director, Computational Sciences Center, AFRL Air Vehicles Directorate, AIAA Fellow. 
The purpose of the present work has been to systematically vary the strength of an oblique shock wave in the Michigan Supersonic Glass Wind Tunnel, and then quantify the sizes and shapes of the various sidewall separation regions. Conditions are varied from "close to unstart" to "far from unstart." Oblique shocks were generated using wedges suspended from the upper wall with angles either six to ten degrees. Separation regions were quantified by recording oil streak patterns on the walls. Shock locations were recorded using a Schlieren system. High-speed video of both the oil streak patterns and the shock wave motions were recorded to understand the time-history of how the separation zones were created during the inlet start-up. Knowledge of this formation sheds light on how the separations later lead to the unsteady unstart of the inlet.

An important aspect of the work is to determine the optimum experimental run conditions. Optimum conditions are defined as those that are best for future CFD modeling as well as for future PIV measurements of the 3-D velocity field. For CFD simulation, it is necessary that a reasonably-sized control volume can be defined that has no reverse flow on either the upstream or downstream boundaries. It was decided that small to moderate separation zones would be best for a first test of CFD models in this demanding 3-D problem. However, the separation zones must be large enough to allow for accurate measurements with PIV, which has a spatial resolution limit. Also, conditions should be "far from unstart" to avoid the possibility that the flow could be unstable. For an inlet that is too close to unstart, small perturbations could lead to undesirable dynamics in either the experiment or the CFD simulation. Additionally, the experimental 3-D flow should be symmetric (separation on the right and left walls should be identical) and as simple as possible. For this reason, the wedge mounted on the top wall was a full-span wedge. Partial-span wedges were previously investigated since they provide less blockage, and provided conditions that were further from unstart. In those cases, the shock became curved before it interacted with the sidewall. This additional complication was avoided by using full span wedges in order to achieve the simplest possible comparison of CFD to the experiment.

The wedge should be suspended from the upper wall because if it is flush mounted, there will be a small recirculation zone in the corner where the upper wall turns at the beginning of the wedge. Thus the shock anchoring can be unsteady, which causes problems when comparing experiment to CFD. With the suspended wedge, the shock is firmly anchored to the sharp leading edge of the wedge. However, it is important that the flow that passes over the top of the suspended wedge be "started." If there is too much blockage of this upper flow, a separation zone can occur between the leading edge of the wedge and the upper wall, which can cause unsteady shock anchoring upstream of the wedge leading edge. To avoid this blockage problem, the upper surface of the wedge is inclined downward.

\section{Experimental Approach}

Experiments were conducted in a vacuum-driven supersonic tunnel with nozzles designed to produce Mach 2.0 or 2.75 free-stream flow for a continuous run time of five to ten minutes. The test section is 2.25 inches wide by 2.75 inches tall and the working fluid consists of conditioned air from the laboratory. Flow straightening is employed upstream of the nozzle to enhance uniformity. In the Mach 2.75 and 2.0 configurations the unit Reynolds number was $8.9 \times 10^{6}[1 / \mathrm{m}]$. Glass sidewalls along the entire length, as well as in a region along the centerline, ensure adequate optical access for all measurements and flow visualization. In all cases below, the experiment was mounted approximately 22 inches $[0.558 \mathrm{~m}]$ from the throat. Figure 1 is a schematic of the tunnel showing variable wedge mounting options.

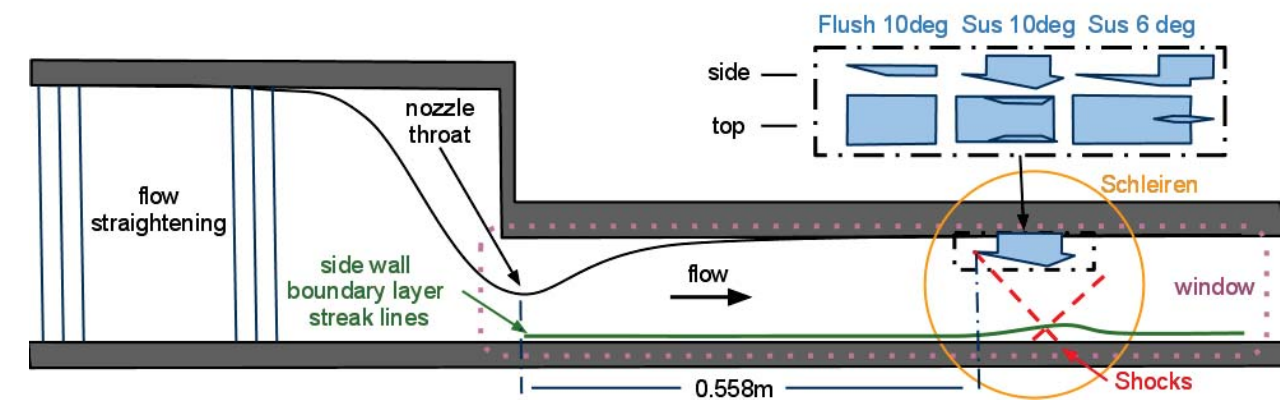

Figure 1. Schematic of the Michigan Glass Wind Tunnel

As seen in Fig. 1, the lower wall of the wind tunnel is flat but it is fed via a bell mouth (not pictured). The tunnel entrance is approximately $200 \mathrm{~cm}$ from the region of interest. Previous SPIV measurements showed that this 
boundary layer (approximately $1 \mathrm{~cm}$ in height) has the Log-Law velocity profile associated with turbulent boundary layers at large Reynolds numbers ${ }^{[16]}$. Several experimental cases are outlined below. The following nomenclature will be used:

- "Full span" indicates the leading edge of the shock generator extends continuously between the two side walls. The shock generator is 2.25 inches wide.

- "Suspended" indicates that the wedge separates the wind tunnel into two flows, a small flow is above and most of the flow is below the wedge. Both flows must remains started. This is accomplished by widening the channel to accommodate both the growth of the boundary layer as well as the side wall supports. Care was taken not to expand this upper flow too aggressively.

- "Flush mounted" indicates the leading edge of the shock generator lies in plane with the top wall.

a)

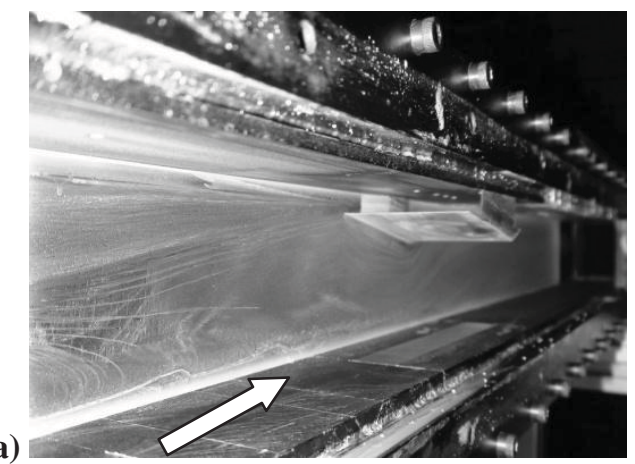

b)

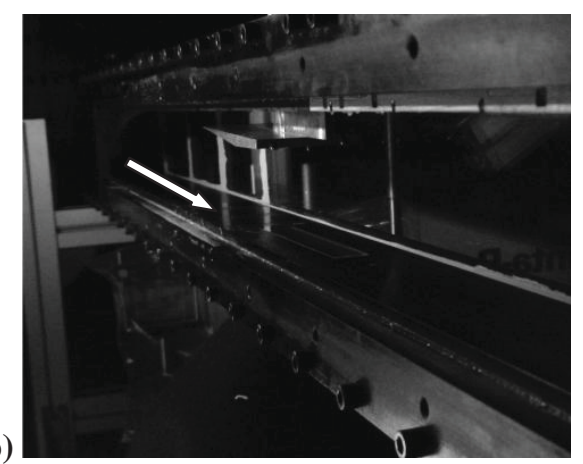

Figure 2. Photographs of the Suspended Shock Generators: (a) Ten Degree (b) Six Degree

Table 1: Test Cases

Case A - Mach 2.75 Full-span suspended 10-degree wedge (Supersonic, but not fully started)

Case B - Mach 2 Full-span suspended 10-degree wedge (Unstarted)

Case C - Mach 2 Full-span flush mounted 10-degree wedge (Upstream shock not anchored)

Case D - Mach 2.75 Full-span flush mounted 10-degree wedge (Anchored but too unsteady)

Case E - Mach 2.75 Full-span suspended 6-degree wedge (Optimum)

With the purpose of exploring corner flows in mind, a full-span wedge geometry was suspended from the top wall of the wind tunnel. As such, an exploratory research program was undertaken as an extension of the CCAS Glass Inlet research program. From the beginning it was understood that large boundary layers would push the experimental geometry to the limits of unstart. One of the four, Case B, did not start and image results for this case have not been included.

\section{A. Visualization methods}

1. Oil Flows

Oil flows ${ }^{[17]}$ are an established method for determining flow separation and the flow topology along 'limiting' surface streamlines, including separation zones. In this case the oil mixture is created by mixing kerosene and calcium carbonate powder in ratios five parts to two by volume. Prior to tunnel operation, a three-inch silicone brush is used to apply the combination to the walls of the wind tunnel perpendicular to the expected direction of flow. Once the valve is opened to start the tunnel, viscous forces begin to drag the solution along the wall. Because kerosene is highly volatile, the low-pressure operating condition at Mach 2.75 rapidly evaporates the oil, leaving behind the calcium carbonate in a streak-like pattern. Convergence of these streak lines occur in the side wall as the swept shock moves down the wall. Oil accumulates in regions of flow separation and does not evaporate as readily. Previous references on oil flow visualization technique describe methods for characterizing this swept shock behavior. Variations in streamline convergence indicate the relative strength of this swept shock boundary layer interaction. In each of the cases, the amount of convergence is used to determine the magnitude of flow separation. 
Photographs were taken of the developing oil flow patterns using a Sony DSC-t70 8.2 Megapixel camera at 720x640 pixel resolution, and video with a Sony HDR-SR12 at 1920x1080 pixel resolution, in both Mach 2 and Mach 2.75 flow arrangements. These videos document the startup transients, the unsteady motion in the recirculation zones, and the relative magnitude of the flow field three dimensionality ${ }^{[18]}$. Still images, as well as individual frames from the video, were analyzed and data was produced to ensure repeatability and compare the effects of varying the Mach number, wedge angle, and shock generator mounting location. Photographs were taken such that scaling/skewing of images was not a significant factor.

\section{Schlieren flow visualization}

A Schlieren system was used to identify shock wave locations. An intensified CCD camera was employed to digitize the images at a 1280x 1024 pixel resolution. The light source was an Oriel Labs 100 watt mercury lamp. Image exposures were feasible down to $25 \mu$ s resolution, but these images had a dynamic contrast of $\sim 2$. Instead images were exposed for $100 \mu \mathrm{s}$, and achieved dynamic contrast of $\sim 10$. At the leading edge of the shock generator, flow deflection angles were measured from the Schlieren images and checked against estimated values based on the Mach number and the $\theta-\beta$-M relations.

\section{Results}

The cases which follow were an exploratory attempt at finding corner flow-shock boundary layer interactions (CFSBLI). Images and videos for each of the five test cases in Table 1 are described sequentially. In each case, the shock boundary layer interaction thickens in the corners, reducing the effective core flow area. As the boundary layer in the corner thickens, its influence reaches upstream, in some cases causing shock waves (sometimes called pseudo-shocks) to originate from these corners. Those shocks further disturb the boundary layer. This feedback

mechanism may be a precursor to unstart. The size of the corner separation varies with swept shock strength and incoming boundary layer size. Larger flow blockage leads to larger separation, eventually producing unstart. Somewhat surprisingly, large-scale three-dimensionality has little effect on traditional Schlieren imaging, but oil flows for the same region clearly show the dominant three-dimensionality in the boundary layer. Simultaneous oil flows on bottom, right, and left walls confirmed flow symmetry about the channel centerline but the documented images below are from only one side-wall.

\section{A. Case A: $M=2.75$ Ten Degree Suspended Wedge}

The Case A conditions in Table 1 produced large-scale sidewall separation zones. Figure 3 shows video image and Schlieren results for Case A. The side-wall interaction spans the entire wall, and the primary sidewall separation zone is 2 inches long [ $5 \mathrm{~cm}$, which is $73 \%$ of the tunnel height]. Converged CFD results from the University of Cincinnati for a similar ten-degree wedge also show a large corner flow recirculation zone. However, CFD simulations do not tend to converge when sufficiently large sidewall separation zones are created; instead the perturbations caused by the unsteady startup may lead to an inlet unstart in the computations.

The Schlieren image for Case A reveals upstream shock wave which causes the shock off the leading edge to skew to nearly forty degrees. The flow deflection from a ten-degree wedge at Mach 2.75 should be thirty degrees. Clearly in this instance the interaction was too strong and too large for useful assessment of CFD. 

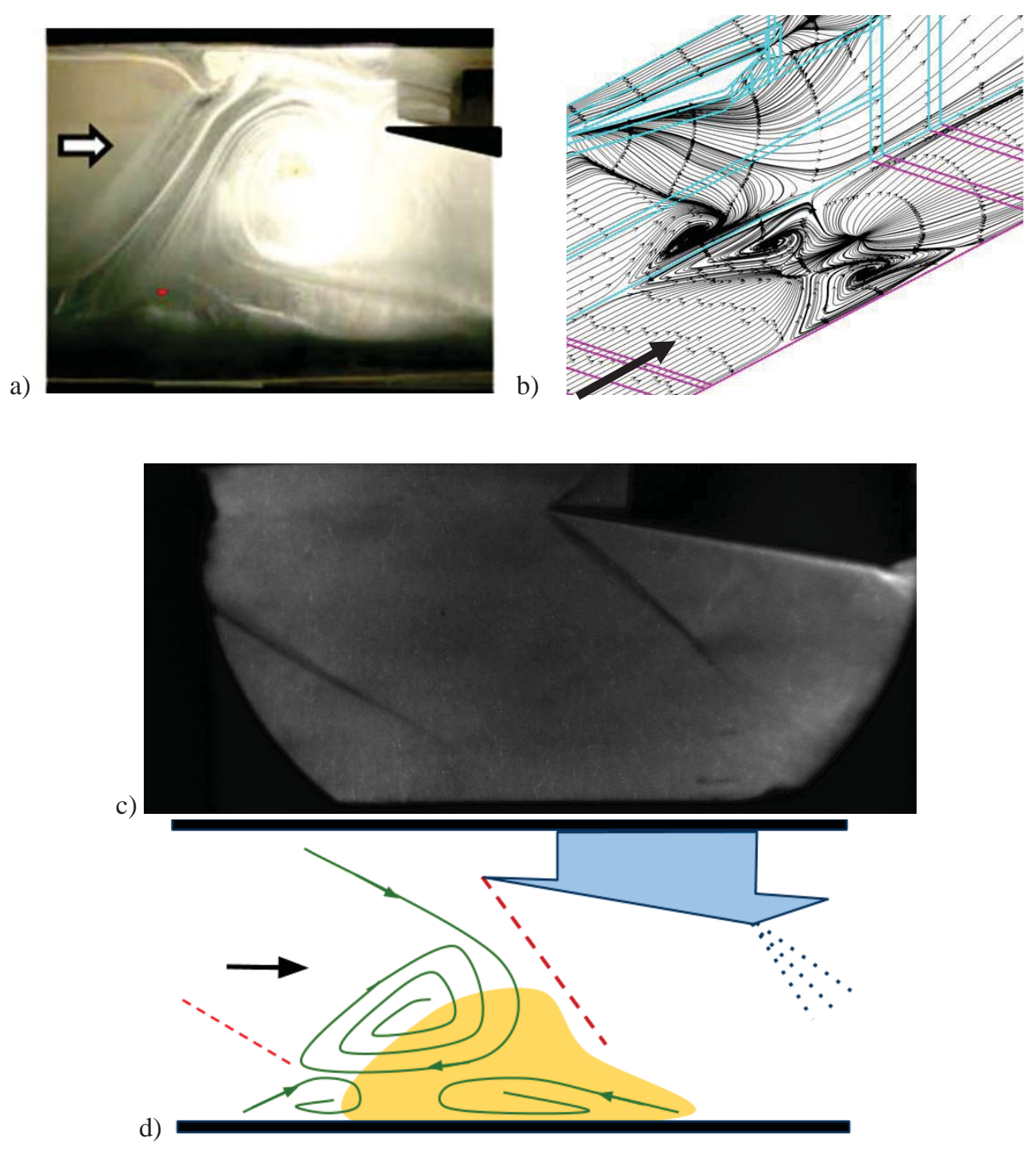

Figure 3. Case A flow patterns caused by a Ten Degree suspended wedge in a Mach 2.75 free stream : a) Oil flow b) CFD 'streak lines' computed at U. of Cincinnati; c) Schlieren image d) Schematic of Interaction

\section{B. Mach $=2.0$ Ten Degree Suspended Wedge}

The previous computational study on the Glass Inlet suggested that lowering the Mach number might reduce the size of the separated zone due to the reduction in the shock strength. However, operating at Mach 2 and using the same wedge geometry resulted in unstart. Therefore no further research for the Case B condition was conducted.

\section{Mach $=2.0$ Ten Degree Flush-Mounted Wedge}

Because a Mach 2 interaction is closer to a cruise flight regime Mach number, the next step was to operate the experiment at Case C, which had a reduced amount of blockage compared to Case B. Using a flush-mounted full span wedge, results in a smaller corner interaction than Case A. However, the upstream motion ahead of the shock generator was undesirable. Oil flow investigation of the region indicated that a CF-SBLI occurs at the top wall, causing unsteadiness in the shock foot region. 


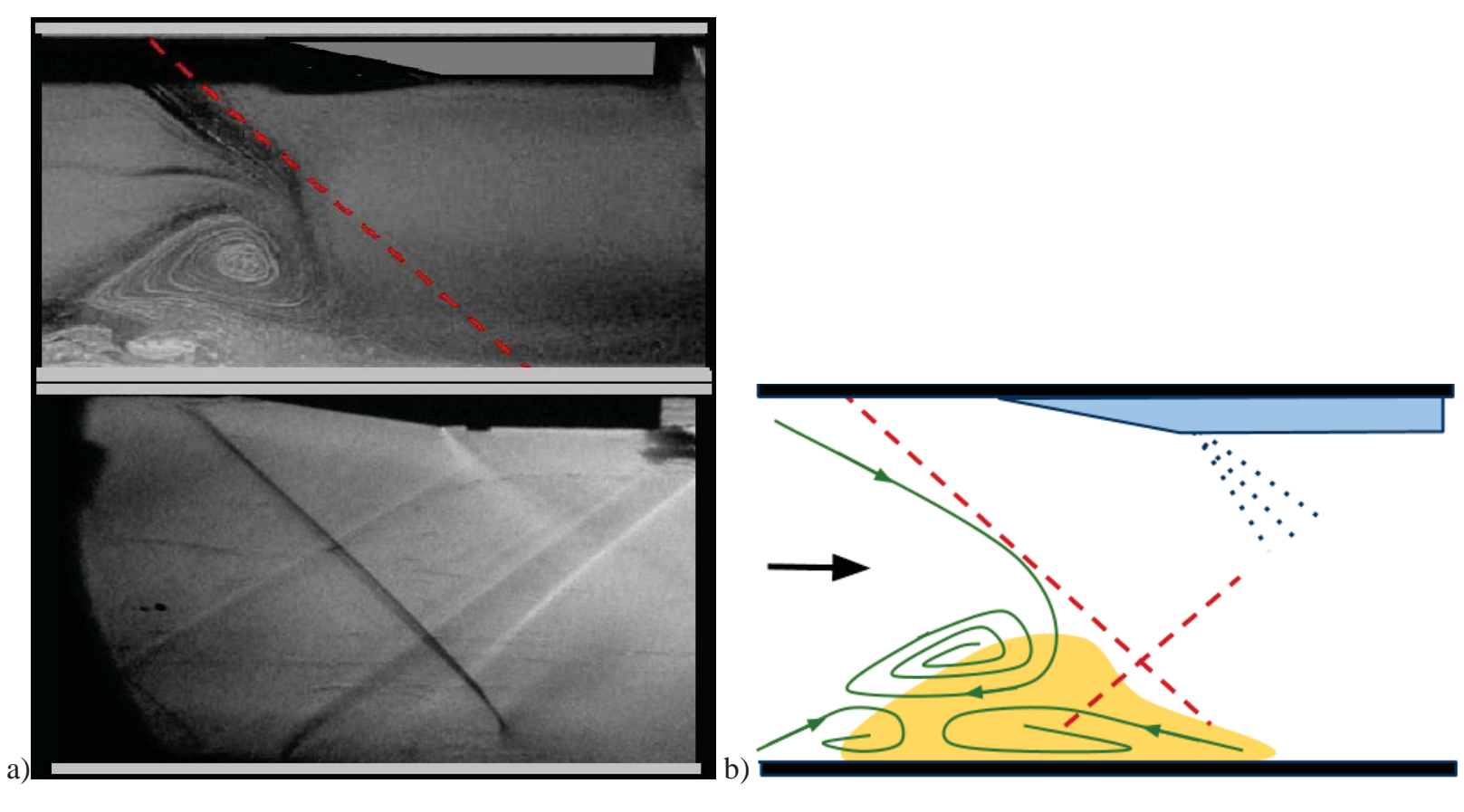

Figure 4. Case C results: Ten Degree Mach 2.0 Flush-mounted wedge a) oil and Schlieren b) schematic of the interaction

a)
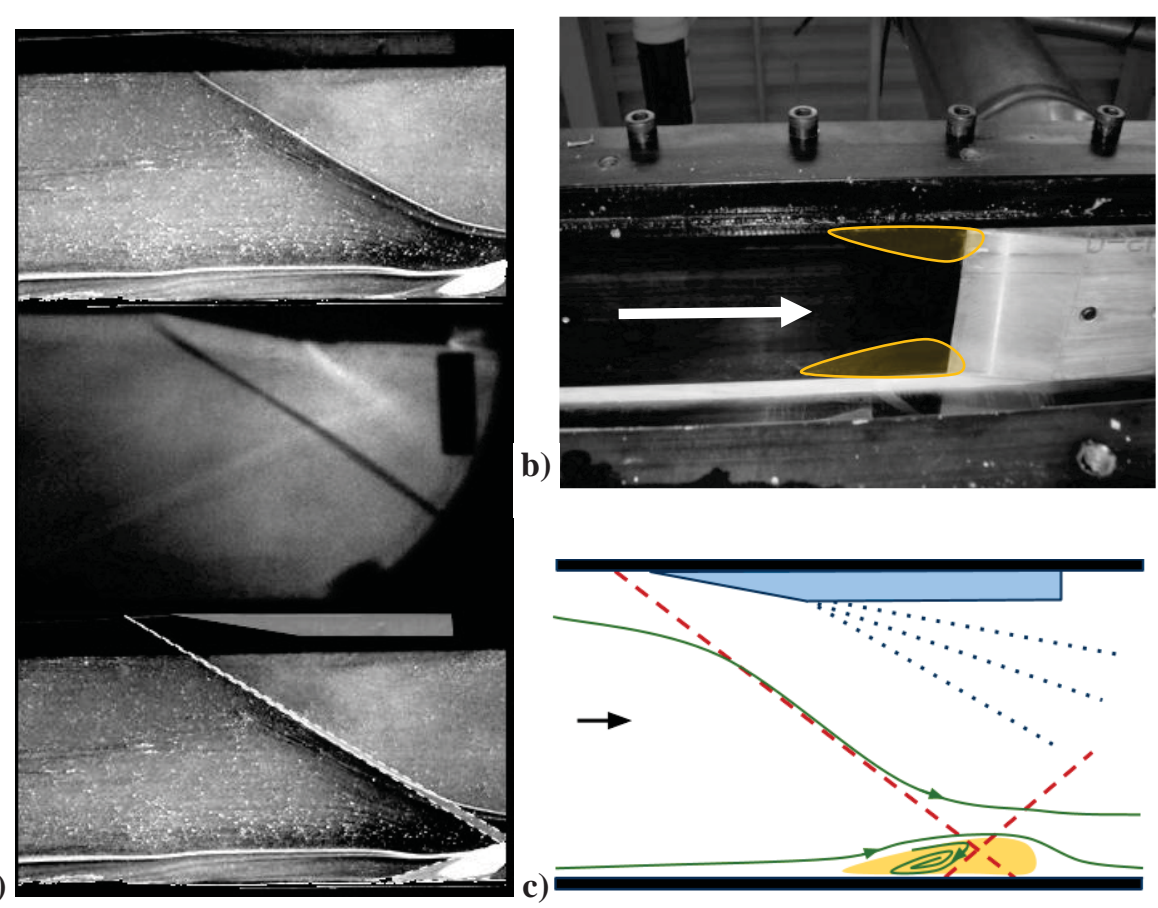

Figure 5. Case D results: Ten Degree Mach 2.75 Flush-Mounted Wedge: a) oil flow and Schlieren visualization b) Top wall CF-SBLI, c) schematic of interaction 


\section{Case D: Mach 2.75 Ten Degree Flush-Mounted Wedge}

In an attempt to anchor the shock wave to the shock generator and avoid unstart, the ten-degree wedge was installed in the Mach 2.75 wind tunnel. Figure 5 shows the attachment was successful. Surprisingly the sidewall separation was reduced despite an increase in shock strength. This somewhat unexpected result can be explained by examining the oil flow pattern. The increase in sweep of the incident shock actually lowers the normal Mach number of the flow along the sidewall, in this case allowing the flow along the wall to turn upstream underneath the shock before reaching the bottom wall.

\section{E. Case E: Mach 2.75 Six Degree Suspended Wedge}

The final test case that provided optimum operating conditions was Case E; it avoided the unstart problems of the previous models by using a free stream Mach number of 2.75, but reduced the shock strength by reducing the flow turning angle. The image in Figure 6 shows that streamlines do not converge under the swept shock for this condition. The corner flow region can still be identified as a separation zone, but is now the optimum size of $2 \mathrm{~cm} \mathrm{x}$ $2 \mathrm{~cm}$. This is sufficiently large for future PIV studies, which have a limited spatial resolution, and is sufficiently small for future CFD simulations.
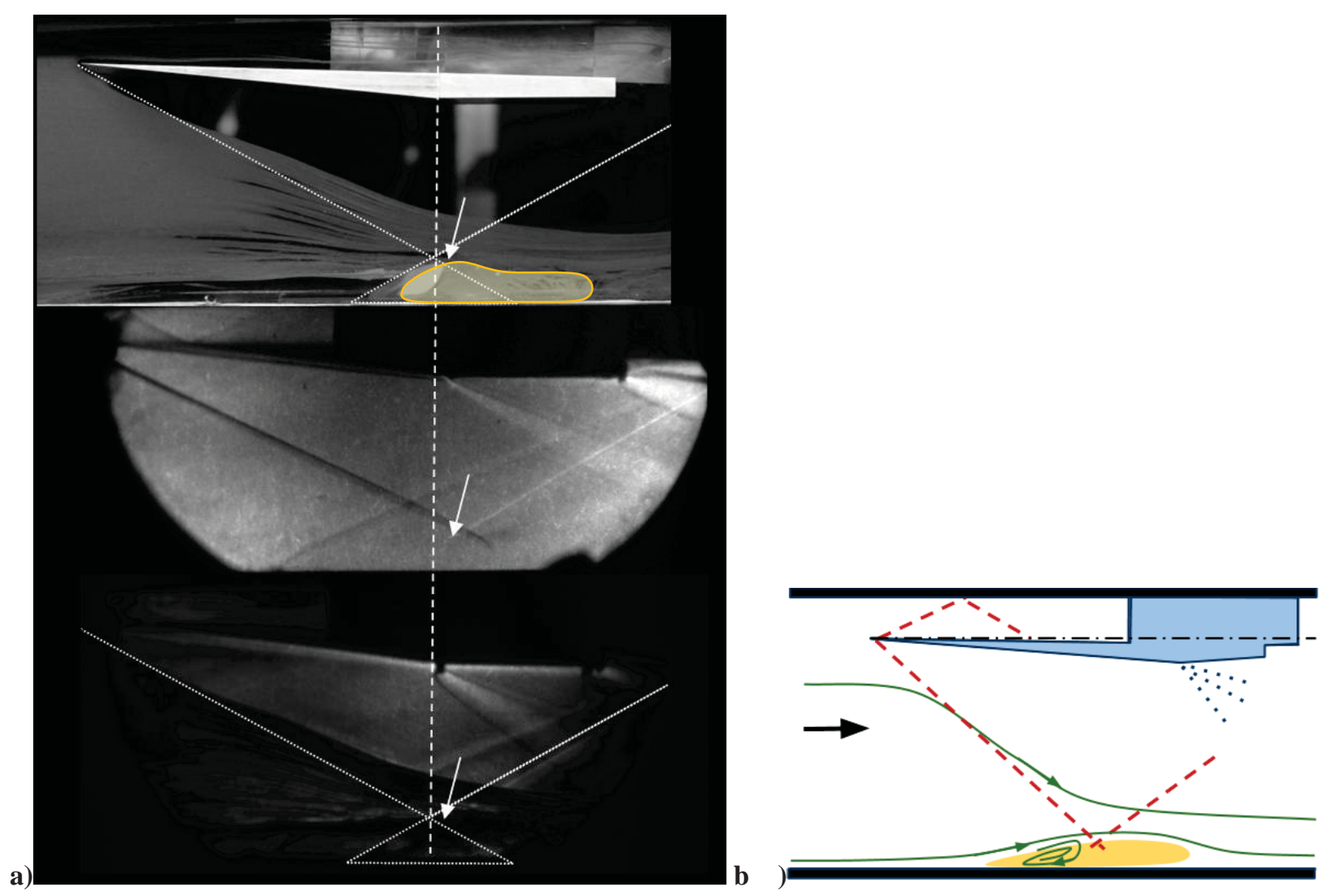

Figure 6. Six Degree Mach 2.75 Suspended Wedge: a) oil and Schlieren visualization b) schematic of the interaction 


\section{F. Centerline Separation Region}

In contrast to the partial span wedges previously investigated, oil flow on the bottom wall at the oblique shock reflection generated by a full span wedge did not indicate flow separation on the centerline. However, downstream of the wedge region, converging wall flow streamlines do produce a small centerline recirculation zone.
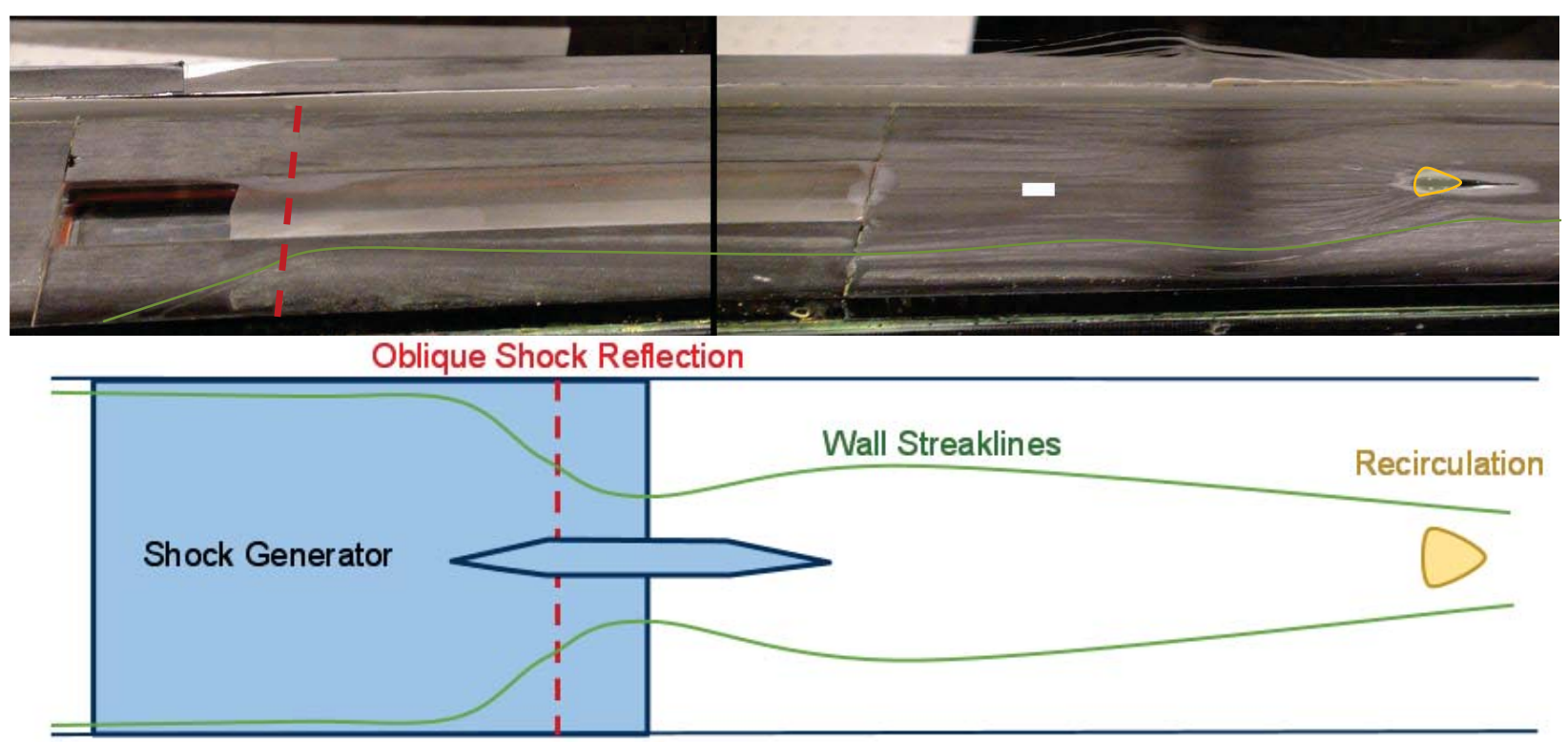

Figure 7. Case E: Bottom wall oil flow

\section{Discussion}

\section{A. CF-SBLI mechanism}

The mechanism of the corner flow SBLI (CF-SBLI) appears to be similar in each of the five cases (A to E) studied. The primary difference is the size of the streak line pattern and its convergence as the bottom wall is approached. As shown in Figs. 8 and 9, the shock from the leading edge sweeps along the side wall, bending of the streaklines indicates weak CF-SBLI flows while convergence of the streaklines leads to strong CF-SBLI. The bending/convergence creates downflow toward the bottom wall boundary layer. Figure 10 diagrams how the flows might interact in the turbulent corner. Reverse flow caused in the side wall by the adverse pressure gradient moves upstream until it reaches a saddle point. For small-scale features, the saddle point is very close to the wall, making it hard to observe. Flow along the bottom wall appears to bend back toward the free stream in the region of shock reflection. The stronger shock creates a more severe secondary flow and a more severe CF-SBLI. 

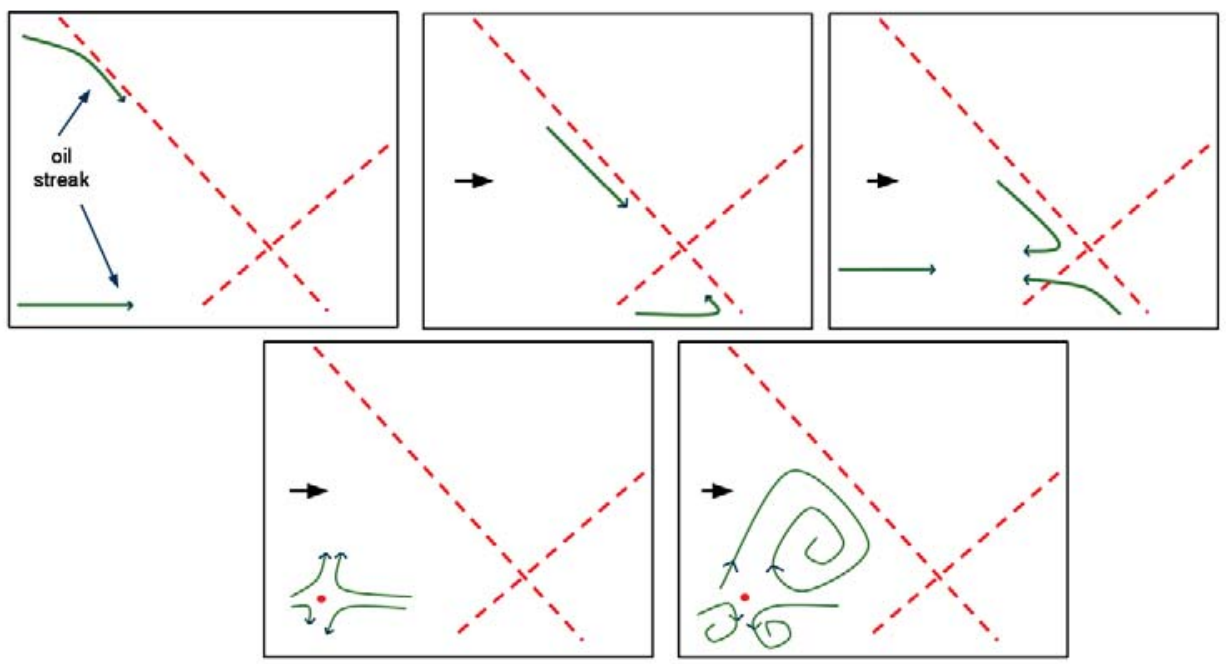

Figure 8. Schematic of the flow deflection near the sidewall, for the Strong CF-SBLI (Case A), based on observed oil patterns.
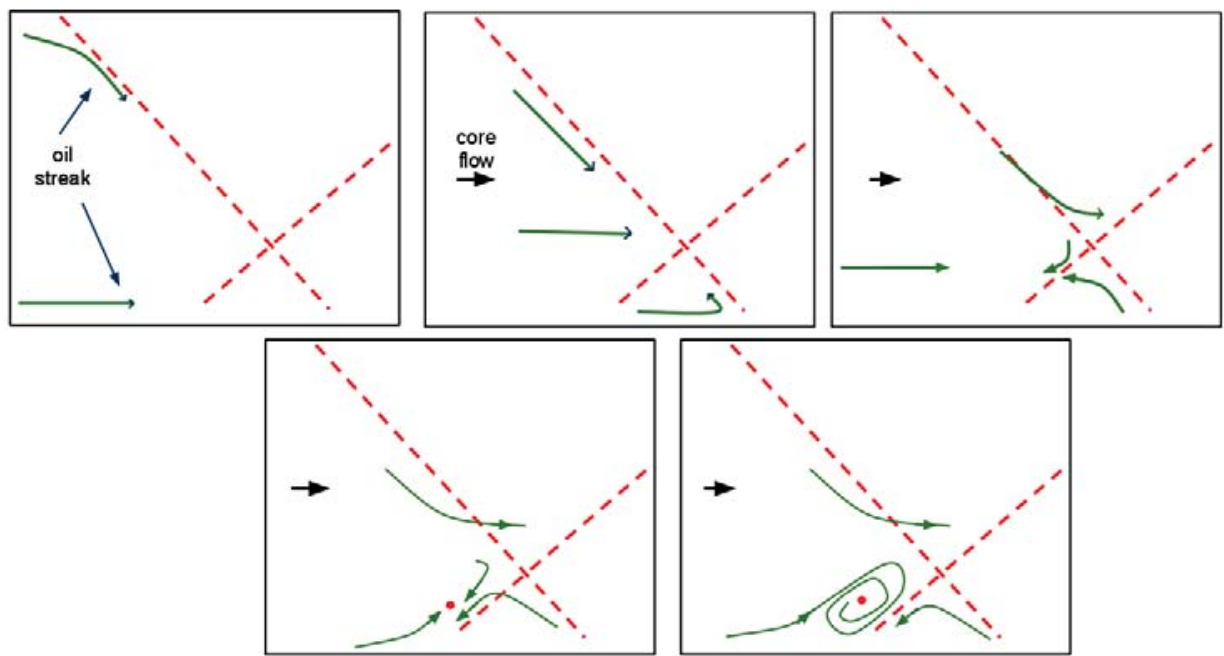

Figure 9. Schematic of the flow deflection near the sidewall, for the Weak CF-SBLI (Case E), based on observed oil patterns.

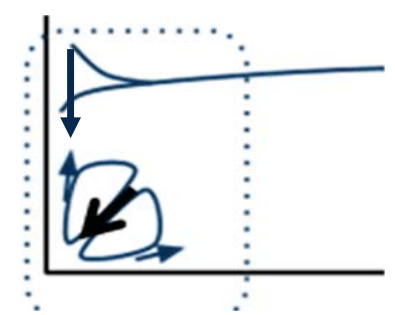

Figure 10. Schematic of Secondary Flows from a Turbulent Corner and Sidewall SBLI 


\section{B. Optimum CF-SBLI research conditions}

Case E, which is a full span 6-degree suspended wedge at Mach 2.75, exhibits all of the desired characteristics for a more thorough combined experimental / CFD study of the CF-SBLI physics. Moderate corner flow separation occurs downstream of the leading edge. Recirculation from the corner does not reach upstream of the leading edge and boundary layer reattachment occurs quickly downstream, creating a suitable outflow boundary condition for CFD. The $2 \mathrm{~cm} \times 2 \mathrm{~cm}$ region is a reasonable control volume for detailed study by the SPIV diagnostics.

The full span wedge is better than the partial span wedge, since the latter produces a curved shock that creates unnecessary complexities. A Mach number of 2.75 is preferred over Mach 2.0 because the latter is too close to inlet unstart. The Mach 2.75 conditions are sufficiently far from unstart that the flow remains steady and impervious to small perturbations. The $10 \mathrm{~mm}$ tall initial boundary layer height provided by the experiment is optimum because it can be probed using the spatial resolution achievable with PIV diagnostics and it is a fully equilibrated boundary layer that has a profile that follows the well established Log Law. The Case E conditions created a CF-SBLI control volume region that is approximately $2 \mathrm{~cm}$ by $2 \mathrm{~cm}$ by $2 \mathrm{~cm}$. This control volume region is optimum for both PIV and CFD future studies.

\section{Acknowledgements}

This work is sponsored by the US Air Force Collaborative Center for Aeronautical Sciences. The authors acknowledge helpful comments from Jon Tinapple and Lewis Suber. The first author especially acknowledges the help in preparing the manuscript provided by Kristin Fraser.

\section{References}

1. Pitt-Ford, C. W., and Babinsky, H. "Micro-Ramp Control for Oblique Shock Wave Boundary Layer Interactions," 2007.

2. Dupont, P., Piponniau, S., Sidorenko, A., and Debive, J. F. "Investigation by Particle Image Velocimetry Measurements of Oblique Shock Reflection with Separation," AIAA Journal Vol. 46, No. 6, 2008, pp. 1365-1370. doi: 10.2514/1.30154

3. Wu, P. P., and Miles, R. B. "Megahertz Visualzation of Compression-Corner Shock Structures," AIAA Journal Vol. 39, No. 8, 2001, pp. 1542-1547.

4. Panaras, A. "Review of the Physics of Swept-Shock/Boundary Layer Interactions," Progress in Aerospace Sciences Vol. 32, 1996, pp. 173-244.

5. Schmisseur, J. D. "An Experimental Study of Fluctuating wall pressures in a highly swept, sharp fininduced mach 5 shock wave / turbulent boundary layer interaction." Wright Patterson, 1993, p. 150.

6. Lu, F. "Upstream influence scaling of Fin-Generated Shock Wave Boundary Layer Interactions," 28th Aerospace Sciences Meeting. AIAA, Reno, NV, 1990.

7. Lu, F., and Settles, G. S. "Color surface-flow visualization of fin-generated shock wave boundary layer interactions," Experiments in Fluids Vol. 8, 1990, p. 3.

8. Handa, T., Masuda, M., and Matsuo, K. "ThreeDimensional Structure of Normal Shock WaveTurbulent Boundary Layer Interaction in a Rectangular Duct by a Laser-Induced Fluorescence Method," Engineering Sciences Reports Vol. 24, No. 2, 2002, pp. 179-185. doi: 10.1007/s001930050092

9. Doerffer, P. "An experimental investigation of the Mach number effect upon a normal shock wave Tubulent boundary layer interaction on a curved wall," Acta Mechanica Vol. 76, No. 1, 1989, pp. 35-51. doi: 10.1007/bf01175795

10. Doerffer, P., and Dallmann, U. "Separation Structures Produced by a Normal Shock-Wave Turbulent Boundary-Layer Interaction in a Narrow Wind Tunnel," 19th Fluid Dynamics, Plasma Dynamics and Lasers Conference. AIAA, Honolulu, Ha, 1987.

11. Clemens, N. T., and Narayanaswamy, V. "Shock Turbulent Boundary Layer Interactions: Review of Recent Work on Sources of Unsteadiness," 38th AIAA Fluid Dynamics Conference. AIAA, San Antonio, TX, 2009, p. 25.

12. Adamson, T. C., and Messiter, A. F. "Analysis of two-dimensional interaction between shock waves and boundary layers," Annual Review of Fluid Mechanics Vol. 12, 1980, p. 35.

13. Green, J. E. "Interactions between shock waves and turbulent boundary layers," Progress in Aerospace Sciences Vol. 11, 1970, pp. 235-260, IN9-IN10, 261-340. doi: Doi: 10.1016/0376-0421(70)90018-7

14. Benek, J. A. "Lessons Learned from the 2010 AIAA Shock Boundary Layer Interaction Workshop," 28th AIAA Applied Aerodynamics Conference. Chicago, IL, 2010, p. 13. 
15. Galbraith, D. S., Orkwis, P. D., Turner, M. G., Galbraith, M. C., Eagle, W. E., and Driscoll, J. F. "Design of a Glass Supersonic Wind Tunnel Experiment for Mixed Compression Inlet Investigations," 40th Fluid Dynamics Conference and Exhibit. Chigago, IL, 2010, p. 17.

16. Lapsa, A. P., and Dahm, W. J. A. "Stereo Particle Image Velocimetry of Nonequilibrium Turbulence Relaxation in a Supersonic Boundary Layer," Experiments in Fluids, 2010.

17. Lu, F. K. "Surface oil flow visualization," The European Physical Journal Special Topics Vol. 182, No. 1, 2010, pp. 51-63. doi: 10.1140/epjst/e2010-01225-0

18. Pierce, A. J., Lu, F. K., Bryant, D. S., and Shih, Y. "New Developments in Surface Oil Flow Visualization," 27th AIAA Aerodynamic Measurement Technology and Ground Testing Conference. AIAA, Chicago, Il, 2010. 